\title{
Human learning in multiple $T$ maze: An investigation of verbal and motor learning modes
}

\author{
William T. Stellwagen and Gerald W. Card \\ MICHIGAN STATE UNIVERSITY
}

\begin{abstract}
Abstraet
Maze learning efficiency was measured under verbal and motor learning modes and single and double maze conditions to investigate additivity of response produced cues. The results indicated that an additivity of response produced cues assumption would not account for superiority of verbal mode maze learners.

\section{Problem}

In learning tasks of the maze type, Ss who verbalize at each choice point tend to reach criterion in fewer trials than those not reporting verbalizations (Husband, 1928; Esper, 1935). This result has been interpreted in terms of the association value of verbal responses relative to manual movements; however, the efficiency of verbal mode learners may be due, in part, to the addition of verbal response produced cues to kinesthesis provided by manual movements. Pooling of the two signal systems with exteroceptive stimulation might produce patterns of stimulation that are more distinctive as well as more massive and this could result in efficient learning through a reduction of choice point generalization.
\end{abstract}

\section{Method}

The mazes used were a variation of the "Stone" raised multiple $\mathrm{T}$ with 20 choice points. Some Ss learned a single maze while others learned two mazes. In the two-maze situation Ss learned, simultaneously, one maze with the left and an identical or mirror image maze with the right hand. Ss were classified into predominantly verbal and predominantly motor mode learners. One group of Ss learned a list of directional English words (up, left, right, down) by the serial anticipation method; these words providing a verbal map of the maze. This condition provided a single signal system control for verbal mode learners. The traditional single maze motor mode learners provided a single signal system control for the other motor learning conditions. The maze used may be inspected in Fig. 1.

Ss in the maze groups were blindfolded and they were instructed to make finger movements in conjunction with an auditory cue, provided by the change mechanism of an automatic slide projector set at a 3-sec. interval. Single maze learning group Ss (SM) were given a brief explanation of the nature of maze learning and after the blindfold was in place, they were allowed to explore a section of the maze. When learning trials were begun errors and the number of trials required to reach a criterion of three perfect trials were recorded. When an error was made Ss were returned to the start position and presented with the next trial. When Ss reached criterion they were asked to describe the method of learning employed. Ss were asked if any method or strategy had been employed with a secondary method discarded. The other maze groups, two identical mazes (IM) and two mirror image mazes (MIM), were exposed to an identical procedure except that these Ss learned, simultaneously, two mazes, one with each hand.

The verbal control group (VC) was instructed to anticipate the list of directional English words, presented in a slide projector at 3-sec. intervals, beginning with a "start"'slide. Upon seeing the start slide $S$ verbally anticipated the word that followed. No cues were given concerning the words or their sequence. Whenever $S$ failed to anticipate a word correctly, the entire list was restored to the start

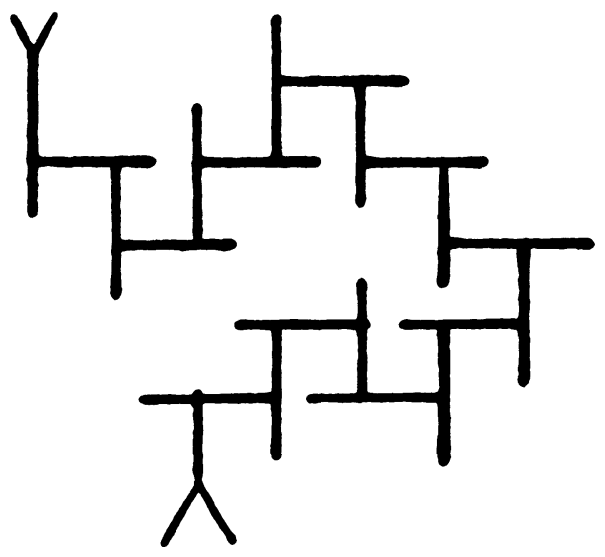

Fig. 1. The Stone Multiple T Maze. Outside dimensions $10 \times 11$ in.

position and $S$ began again. The verbal series taken from the single maze was "'Right, Up, Right, Down, Right, Up, Right, Up, Left, Up, Left, Up, Left, Down, Left, Up, Left, Up." Criterion was three perfect trials.

Seventy Ss from undergraduate psychology classes served on a volunteer basis. Ten Ss were assigned to group VC, 20 to SM, 20 to IM, and 20 to MIM.

\section{Results}

After-the-fact questioning produced four learning categories, verbal, motor, verbal in the later trials, and visual imagery. Ss reporting visual imagery were few in number and they were discarded. The verbal-motor mode classification and various maze learning conditions produced unequal cell $n$ 's, thus it appeared that the learning conditions variable was a determinant of the number of Ss adopting the learning modes, $x^{2}=10.6 ; \mathrm{df}, 2 ; \mathrm{p}<.01$. By randomly discarding two Ss from VC, two from SM motor, nine from IM verbal, four from MIM motor, and pooling Ss reporting verbal and verbal in later trials categories in SM verbal, equal n's of eight were obtained for all groups except IM motor which contained only three Ss. The latter group was dropped from preliminary analysis.

An analysis of variance of the trials to criterion scores for the verbal and motor learning modes exposed to the several learning conditions yielded a highly significant $\mathrm{F}$, df, 5 and $47 ; \mathrm{F}=17.04 ; \mathrm{p}<.001$. There were no significant differences when individual comparisons between the verbal control group and the various verbal mode maze learning groups were made with Dunnet's procedure for comparing $\mathbf{n}$ means with a control; VC vs. SM verbal, $d f, 14 ; t=.36 ; n$. s.; VC vs. 
Table 1. Means and Standard Deviations of the Trials to Criterion Scores

\begin{tabular}{|c|c|c|c|c|c|c|c|c|}
\hline Learning Mode & Verbal Contro & (VC) & Single Maze & (SM) & Identical Mazes & (IM) & Mirror Image & (MIM) \\
\hline & $M$ & SD & M & SD & M & SD & M & SD \\
\hline Verbal & $21.30(n=10)$ & 23.70 & $21.33(n=6)$ & 5.35 & $27.88(n=17)$ & 5.62 & $29.63(n=8)$ & 5.41 \\
\hline Verbal Later Trials & $-\cdots$ & $-\ldots$ & $26.25(n=4)$ & 6.41 & $-\cdots$ & --- & $-\cdots$ & --- \\
\hline Motor & $-\ldots$ & $-\cdots$ & $40.50(n=10)$ & 11.46 & $83.67(n=3)$ & 5.31 & $64.83(n=12)$ & 14.22 \\
\hline Unclassified (Visual) & $-\cdots$ & $-\ldots-$ & $22.50(n=2)$ & 2.50 & --- & --- & ---- & $-\cdots$ \\
\hline Pooled Verbal & $22.38(n=8)$ & 8.37 & $25.13(n=8)$ & 5.35 & $26.88(n=8)$ & 5.62 & $29.63(n=8)$ & 5.41 \\
\hline Pooled Motor & - & $-\ldots$ & $40.88(n=8)$ & 11.46 & $83.67(n=3)$ & 17.94 & $59.38(n=8)$ & 14.21 \\
\hline
\end{tabular}

IM verbal, df, $14 ; t=.93 ; n$. s.; and VC vs. MIM verbal, df, $14 ; t=1.49 ; n$. $s$.

When individual comparisons between the single signal system control (SM motor) and the other motor modes were made with Dunnet's $t$, both possible comparisons were significant; SM motor vs. MIM motor, df, 14; $t=8.06 ; p<.005$; and SM motor vs. IM motor, $\mathrm{df}, 9 ; \mathrm{t}=2.75 ; \mathrm{p}<.05$. The latter comparison must be cautiously interpreted because of the small $\mathrm{n}$ involved. In addition, the pooled verbal vs. pooled motor comparison was highly significant, $\mathrm{df}, 41 ; \mathrm{t}=9.54 ; \mathrm{p}<.005$.

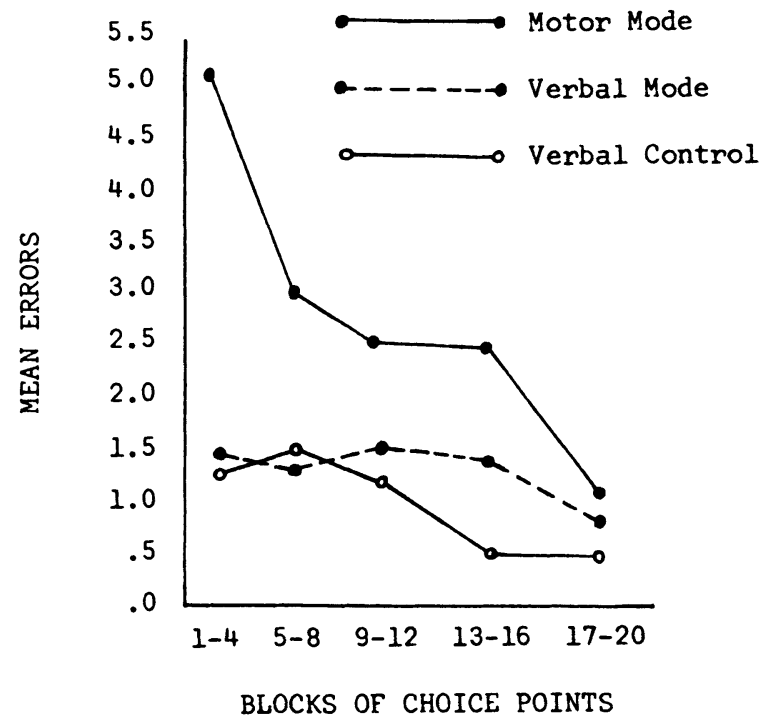

Fig. 2. Serial position curves for pooled motor, pooled verbal, and the verbal control.
The error term for equal $n$ comparisons was taken directly from the analysis of variance, while it was computed separately for comparisons involving IM motor when $\mathrm{n}$ equaled three. There was no evidence of variance heterogeniety in the comparisons reported. The method of error correction used destroyed the usual serial position curves.

\section{Diseussion}

The addition of identical and mirror image mazes did not appear to affect the efficiency of verbal mode learners, while it did affect Ss operating with the motor mode to their decrement. For this reason the verbal control group did not assist the interpretation of results, nor did an additivity of response produced cues hypothesis predict the experimental outcomes. Although further data are needed before interpretation of these results is possible, inspection of the tasks suggests a number of hypotheses. First, verbal mode Ss may have found the task half as long as motor mode Ss since they could learn "up-right, down-left," etc. sequences as pairs. Second, use of a verbal code may have isolated the task removing associative interference from spatial cues, or third, the verbal code may have allowed Ss to focus on self stimulation (meaningfulness or associations of a personal nature). On the other hand, since the tasks involved chaining to an important degree, it may be that differential chaining rates exist for motor as opposed to verbal response produced cues in human Ss.

\section{References}

ESPER, F. A. Language. In C. Murchison (Ed.), A handbook of social. psychology. Worcester: Clark University Press, 1935. Pp. 417-460.

HUSBAND, R. W. Human learning on a four section, elevated finger maze. J. gen. Psychol., 1928, 1, 15-28. 